\title{
Different Work Regimes of an Organic Thin Film Transistor OTFT and Possible Applications in Bioelectronics
}

\author{
Cristian Ravariu ${ }^{1, *}$, Daniela Dragomirescu ${ }^{2}$ \\ ${ }^{1}$ Dept. of Electron Devices, Faculty of Electronics, Polytechnic University of Bucharest, BioNEC Group, Bucharest, Romania \\ ${ }^{2}$ CNRS LAAS, UPS, INSA, INP, ISAE, University of Toulouse, 7 avenue du colonel Roche, Toulouse, France
}

Email address:

cristian.ravariu@upb.ro (C. Ravariu),daniela.dragomirescu@laas.fr (D. Dragomirescu)

\section{To cite this article:}

Cristian Ravariu, Daniela Dragomirescu. Different Work Regimes of an Organic Thin Film Transistor OTFT and Possible Applications in Bioelectronics. American Journal of Bioscience and Bioengineering. Special Issue: Bio-electronics: Biosensors, Biomedical Signal Processing, and Organic Engineering. Vol. 3, No. 3-1, 2015, pp. 7-13. doi: 10.11648/j.bio.s.2015030301.12

\begin{abstract}
This paper presents an organic semiconductor transistor, with a vertical current modulation and a horizontal conduction. The simulations show a stronger top gate influence and establish four work regimes, depending on the top and bottom gates biasing. In the most favorable regime for the holes channel, under the reverse biased $\mathrm{n}^{+} \mathrm{p}$ junction, the holes/electrons current densities ratio reaches 0.168/269. However, an ambipolar OTFT function occurs under the reverse biasing of the vertical junction, with a top n-layer and a bottom p-layer. Due to the asymmetrical doping profile, the $\mathrm{n}^{+}$channel conduction prevails in all the regimes. Therefore, the maximum current density of $1900 \mathrm{~A} / \mathrm{cm}^{2}$ is ensured by a double $\mathrm{n}$ channel, when both gates are positive biased. After simulations, three distinct work regimes are revealed by this single device: a SOI behavior with volume channel, a JFET with neutral median channel and an OTFT with one or more interface channels.
\end{abstract}

Keywords: Organic Transistors, Simulations, Bioelectronics

\section{Introduction}

The organic materials technology offers simple methods of the device manufacturing, including inkjet printing, roll-to-roll printing or non-hydrolytic sol-gel process that promise very low cost electronic products against the lithography methods in silicon technology [1-3].

In spite of the relatively rapid progress of new organic fabrication processes [4], an Organic Thin Film Transistor (OTFT) with reasonable electronic characteristics rests a serious challenge, [5].

For instance, Organic MEtal Semiconductor Field Effect Transistor, OMESFET was proposed as alternatives to OTFT. OMESFETs operate at lower voltages up to $5 \mathrm{~V}$, with better ON/OFF current ratios than an OTFT, but still use only a p-type conduction, [6]. The classical technologies used in the Pentacene OTFT manufacturing, frequently appeal to p-type conduction, except for a few special low band-gap organic semiconductors. Some authors claim that n-type conduction can be accomplished either in special organic gate dielectrics [7], either in special low band-gap organic semiconductors, where the electrons mobility can reaches $0.01 \mathrm{~cm}^{2} / \mathrm{Vs}$, [8].

The main Organic-TFTs are produced in p-type semiconductor. The n-type conduction can be accomplished either in special low band-gap organic semiconductors, [8]. An additional reason for the n-type organic-TFT avoiding is the electrons trapping at the semiconductor-oxide interface by hydroxyl groups, existent as silanols in the usual $\mathrm{SiO}_{2}$ dielectric. OTFTs with pentacene deposited onto a polymer layer enhanced the drain current excursion from $8 \mathrm{nA}$ to $80 \mathrm{nA}$ at the same gate voltage biasing, due to the interface traps optimization, [9].

In this paper, the polyimide organic insulator is considered as the back insulator and the oxide as the front insulator, for the proposed Organic-TFT. The compatibility between the circuit integration technology and the polyimide processing was proved, [10]. The expected results concern a differentiated current capability versus the work regime and alternative functions for the proposed thin film organic transistor.

\section{The Organic-TFT Concept}

Our Organic field effect transistor is related to the Semiconductor on insulator SOI configuration [11] and also corresponds to the Organic Thin-Film Transistors (OTFT), because it uses sub-100nm organic films. Therefore, the back 
and front gates from SOI architecture [12] are re-named here as bottom and top gates, accordingly to the TFT terminology, $[6,7]$.

The proposed device is depicted in Atlas software, fig. 1. The structure is able to collect the accumulation currents from the superior and inferior organic layers, near oxide and respectively polyimide. In this way, an ambipolar conduction by an upper $\mathrm{n}^{+}$channel and a bottom $\mathrm{p}$ channel is possible. However, the metallic shortcut of the vertical $n^{+} p$ junction is in agreement with the zero current condition on the global vertical direction, between the upper oxide and bottom polyimide.

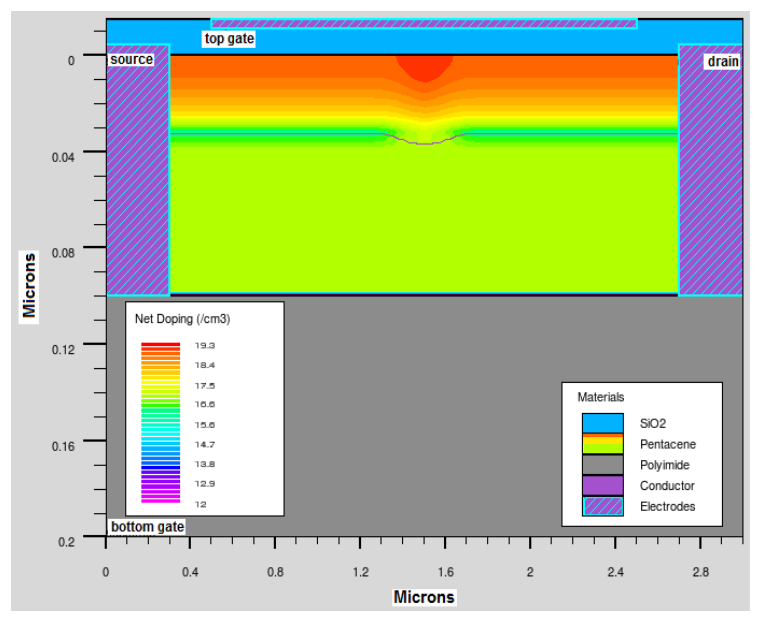

Figure 1. Materials and doping profile within the O-TFT.

The device is depicted in the Atlas software, using a Gaussian profile for the $\mathrm{n}^{+}$doping concentration, assuming a non-uniform profile in the upper semiconductor layer, fig. 1. The organic semiconductor is either the default pentacene from the Atlas library [13] as Pentacene, or optimized organic semiconductors from literature, [14] as Organic new.

The hole and electron mobilities of the reported ambipolar organic semiconductors are typically $0.1 \mathrm{~cm}^{2} / \mathrm{Vs}$, [15]. In order to make possible an ambipolar charge transport, the HOMO (highest occupied molecular orbital) and LUMO (lowest unoccupied molecular orbital) energy levels must be positioned as close as possible and the gate dielectrics must be selected in order to suppress the electron trapping at the organic-dielectric interface, [16]. Consequently, in the simulator, the organic semiconductor / polyimide interface is defined as a thermionic tunnel interface, while the upper oxide / organic semiconductor interface is depicted by a charged interface. A special attention has to be paid to the metallic contacts. Burgi et al. demonstrated that the work function of gold $(5.1 \mathrm{eV})$ is well aligned with the HOMO level of P3HT $(4.8 \mathrm{eV})$, which offers very low contact resistance, while no charge injection occurs for aluminum $(4.0 \mathrm{eV})$, which offers a contact resistance with several orders of magnitude higher, [17].

For our structure, the aluminum contacts offers Schottky barrier with $4.2 \mathrm{~V}$ potential distribution at zero external biasing Also, the simulations reveal a contact resistance dependence on the doping concentrations in the $\mathrm{n}^{+}$or $\mathrm{p}$ type layers.
However, the contact resistances are depending on the gates biasing, due to the carriers concentration modulation under variable gate voltages, [14].

The particular physical features of the standard pentacene, as Pentacene [13], enhanced new organic semiconductors as Organic_new [14, 18] and polyimide insulator [19] are included in the Atlas models by the material statement and centralized in table 1 . Those parameters that are missing from table 1, for pentacene, organic or insulators, are implicitly included in the Atlas library [13]. The sizes and doping of each layers are selected from a real organic technology [10-20], accordingly with the indicated references from table 1 . The $\mathrm{n}^{+}$ type layer has a Gaussian doping concentration with a peak of $10^{19} \mathrm{~cm}^{-3}$, while the substrate $\mathrm{p}$-type has $10^{17} \mathrm{~cm}^{-3}$.

Table 1. Device characteristics

\begin{tabular}{|c|c|c|c|}
\hline Material & Thickness $(\mu \mathrm{m})$ & $\mathbf{E}_{\mathrm{G}}(\mathrm{eV})$ & Mobility $\left(\mathrm{cm}^{2} / \mathrm{Vs}\right)$ \\
\hline n-Pentacene & $40^{[14]}$ & $1.08^{[20]}$ & $2 \times 10^{-5,[20]}$ \\
\hline p-Pentacene & $60^{[9]}$ & $1.08^{[20]}$ & $4 \times 10^{-4,[20]}$ \\
\hline n-Organic_new & $40^{[14]}$ & $1.2^{[18]}$ & $0.50^{,[14]}$ \\
\hline p-Organic_new & $60^{[9]}$ & $1.2^{[18]}$ & $0.53^{,[14]}$ \\
\hline
\end{tabular}

\section{Simulations of the Device Physics}

In order to simulate the organic semiconductor material, the following parameters are included in the model statement: pfmob singlet langevin, in order to activate the Poole-Frenkel mobility model and the Langevin recombination model for the carriers continuity equation, [13].

In the usual Atlas simulations, the drain currents are expressed in Amperes on a transversal depth of $1 \mu \mathrm{m}$. It is expected that in experimental applications with few millimeters depth, the current is usually higher. Therefore, in these simulations the current densities are more relevant than the currents.

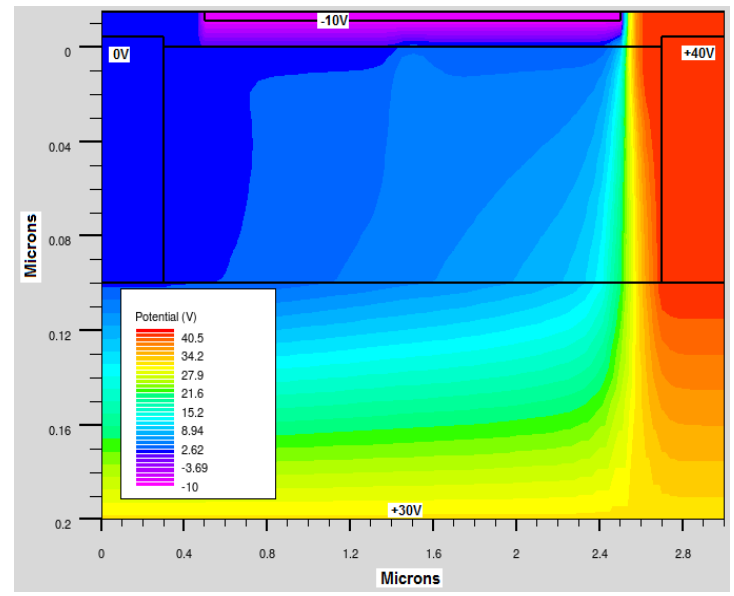

Figure 2. The potential distribution at $V_{S}=0 \mathrm{~V}, V_{D}=40 \mathrm{~V}, V_{T G}=-10 \mathrm{~V}$ and $V_{B G}=30 \mathrm{~V}$.

The potential distribution is available in fig. 2, accordingly with an expecting work regime, for positive drain voltage and negative top-gate voltage. 
The maximum electric field occurs on a $0.2 \mu \mathrm{m}$ length of oxide, between the edges of top gate and drain electrodes. Hence, the maximum electric field reaches $2.5 \times 10^{6} \mathrm{~V} / \mathrm{cm}$, still lower than $1.1 \times 10^{7} \mathrm{~V} / \mathrm{cm}$, but close to the critical electric field in the right corner of the oxide, near the drain contact. Therefore, $+40 \mathrm{~V} \ldots+50 \mathrm{~V}$ could be a safe limit for the drain voltage. The thicker polyimide offers lower leakage currents thru polyimide versus oxide, at these voltages. However, the top and bottom gate current densities are some order of magnitudes lower than the useful longitudinal current, so far away from the breakdown conditions.

The structure comprises a vertical $\mathrm{n}^{+} \mathrm{p}$ junction. Therefore, two types of carriers ensure the longitudinal conduction within OTFT, as in an ambipolar device. A negative bottom gate voltage systematically depresses the electron concentration near the polyimide interface from $10^{11} \mathrm{~cm}^{-3}$ for $\mathrm{V}_{\mathrm{BG}}=0 \mathrm{~V}$, up to $10^{0} \mathrm{~cm}^{-3}$ for $\mathrm{V}_{\mathrm{BG}}=-20 \mathrm{~V}$, but may reinforce the holes concentration.

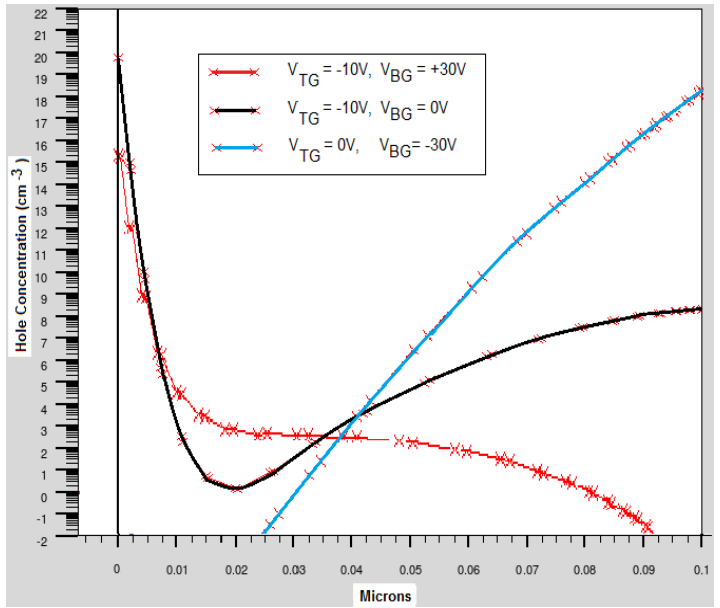

Figure 3. The holes concentration for different top and bottom gate voltages.

A negative top gate voltage can be favorable for the holes accumulation. An additional negative bottom gate voltage enhances the holes concentration, while positive bottom gate voltage depletes the p-type film, fig. 3. Holes accumulation occurs near the polyimide interface for $\mathrm{V}_{\mathrm{BG}}=-30 \mathrm{~V}$, up to $2 \times 10^{18} \mathrm{~cm}^{-3}$, higher than the native doping concentration of $10^{17} \mathrm{~cm}^{-3}$, while holes accumulation occurs near the oxide interface for $\mathrm{V}_{\mathrm{TG}}=-10 \mathrm{~V}$, up to $9 \times 10^{19} \mathrm{~cm}^{-3}$. Hence, the volume n-channel co-exists with upper interface p-channel, especially in the source vicinity. The work regime with positive top gate voltage provides two conduction channels, near oxide and polyimide interfaces, depending on the bottom gate biasing.

Figure 4 presents in doted lines the electrons concentration and in solid lines the holes concentration, under the gates action at $\mathrm{V}_{\mathrm{TG}}=+1 \div+10 \mathrm{~V}$ and $\mathrm{V}_{\mathrm{BG}}=-20 \mathrm{~V} \div+30 \mathrm{~V}$. For the positive top gate voltage, a negative bottom voltage only reduces the electron channel width, accompanied by holes accumulation channel developing at the organic semiconductor / polyimide interface. A positive bottom voltage attracts a second electron channel near the polyimide interface, keeping away the holes accumulation, under $10^{-8} \mathrm{~cm}^{-3}$. In this last case, both $\mathrm{n}$-channels have the electrons reservoir in the $\mathrm{n}^{+}$-type region, with high electrons concentration in the entire organic semiconductor $-10^{17} \div 10^{19}$ $\mathrm{cm}^{-3}$, and two peaks of $10^{20} \mathrm{~cm}^{-3}$ near the oxide and, respectively $5 \times 10^{19} \mathrm{~cm}^{-3}$, near the polyimide interface. Additionally, the electrons-holes recombination processes are insignificant, in this case too. Consequently, this work regime is suspected to offer the maximum current capability.

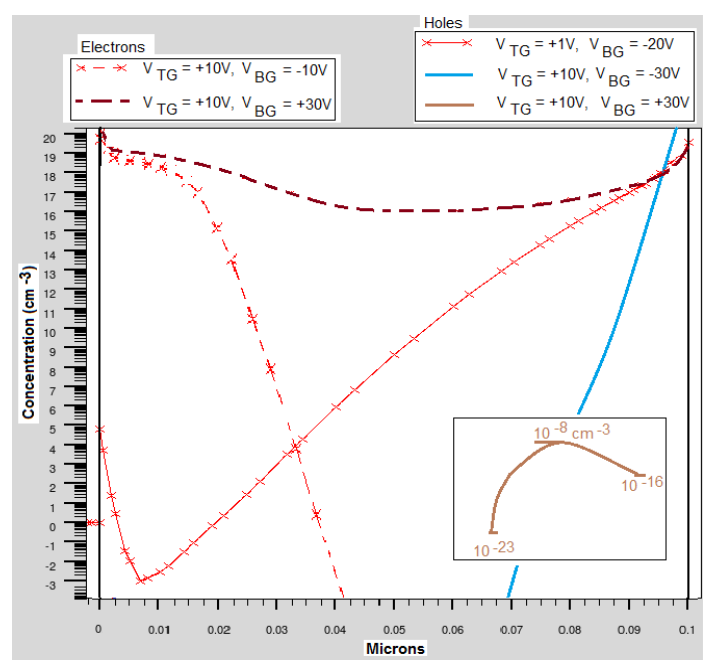

Figure 4. The electron and hole concentrations at positive top gate voltages.

\section{Static Regime Analysis}

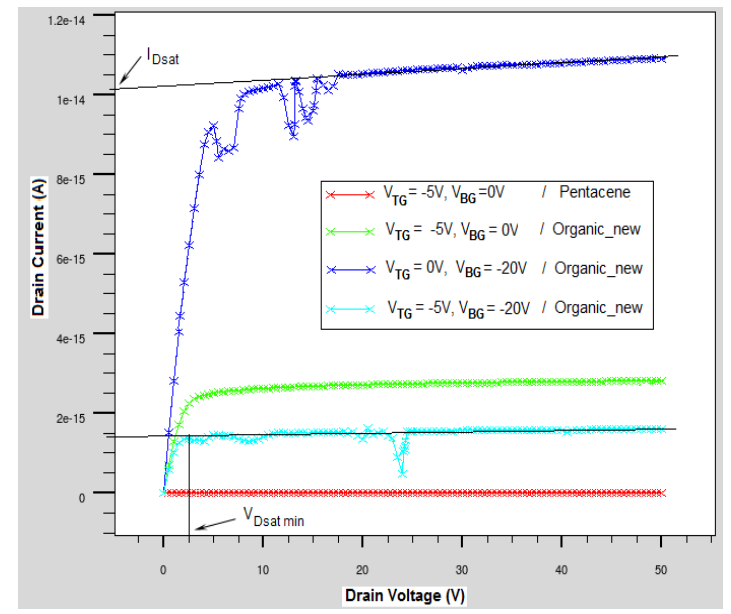

Figure 5. The output characteristics at negative top gate voltage, for new and old organic semiconductors.

First simulations are focused on the output characteristics, $\mathrm{I}_{\mathrm{D}}-\mathrm{V}_{\mathrm{DS}}$, at negative top gate voltage, corresponding to the electron depletion mode, fig. 5. The implicit pentacene material, from the Atlas library (Pentacene), offers extremely low drain currents around $\mathrm{I}_{\text {Dsat }}=10^{-18} \mathrm{~A}$, due to the poor mobility. Therefore in next simulations, only the organic new is considered, as organic semiconductor. The maximum drain current $\mathrm{I}_{\text {Dsat }}=10.7 \mathrm{fA}$ occurs for $\mathrm{V}_{\mathrm{BG}}=-20 \mathrm{~V}, \mathrm{~V}_{\mathrm{TG}}=0 \mathrm{~V}$, fig. 5 . The current decreases when the negative top or bottom voltages increase in modulus, due to the n-channel depletion.

(Space here ??)

In a second stage, the output characteristics are 
comparatively simulated at different top and bottom gate voltages, only for Organic_new material, fig. 6 .

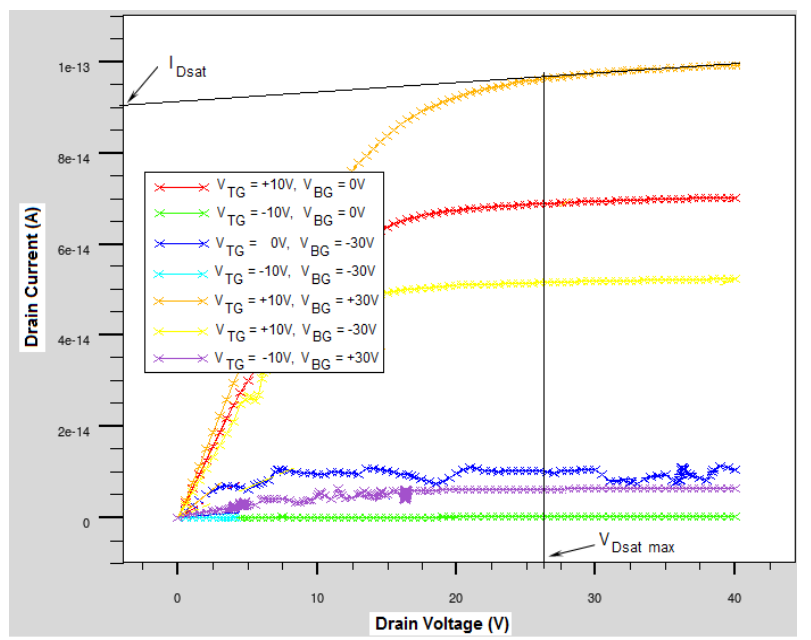

Figure 6. The output characteristics at different top and bottom gate voltages.

The higher positive gate voltages stimulate higher drain currents. The maximum saturated drain current of $100 \mathrm{fA}$ occurs for $\mathrm{V}_{\mathrm{TG}}=+10 \mathrm{~V}, \mathrm{~V}_{\mathrm{BG}}=+30 \mathrm{~V}$. If the top gate voltage is maintained positive, the current is kept in a higher range between 50-100fA, with a poor dependence on the bottom gate voltage. A drain current decreases with one order of magnitude, under a negative top gate voltage. This behavior is caused by the asymmetrical doping concentration inside the initial $\mathrm{n}^{+} \mathrm{p}$ junction.

\section{Simulations of the Dynamic Aspects}

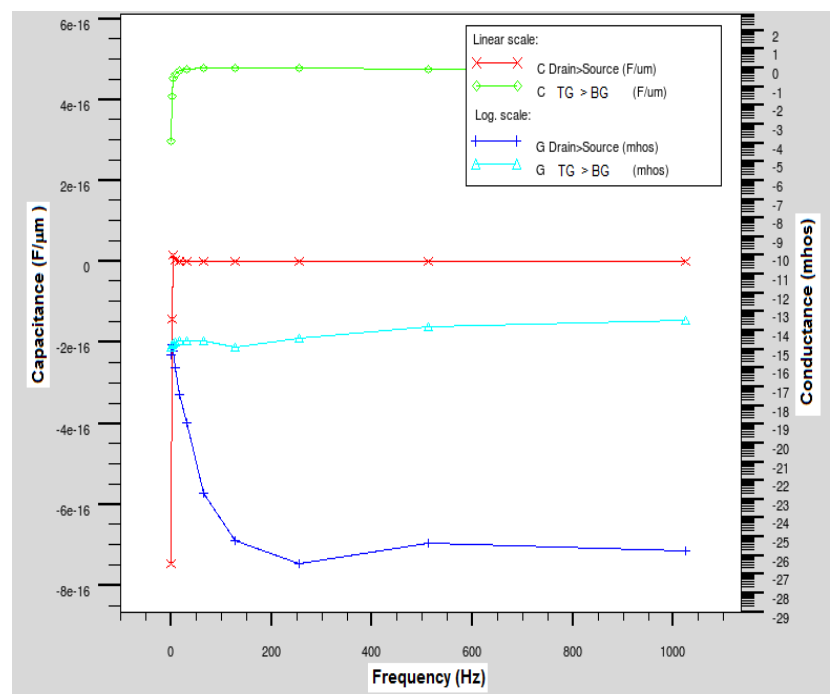

Figure 7. The dynamic curves $C_{T G-B G}, G_{T G-B G}$ and $C_{D S}, G_{D S}$ versus the frequency.

In the first analysis, the capacitances and conductances are simulated against the frequency. The cross-sections on longitudinal and transversal direction occur between Top Gate - Bottom Gate, respectively Drain - Source electrodes. The proposed device is simulated when the frequency increases from $0.1 \mathrm{~Hz}$ to $1 \mathrm{kHz}$ on the top gate terminal, while the DC signals are: $\mathrm{V}_{\mathrm{S}}=0 \mathrm{~V}, \mathrm{~V}_{\mathrm{D}}=20 \mathrm{~V}, \mathrm{~V}_{\mathrm{BG}}=-5 \mathrm{~V}, \mathrm{~V}_{\mathrm{TG}}=+10 \mathrm{~V}$, fig. 7 .

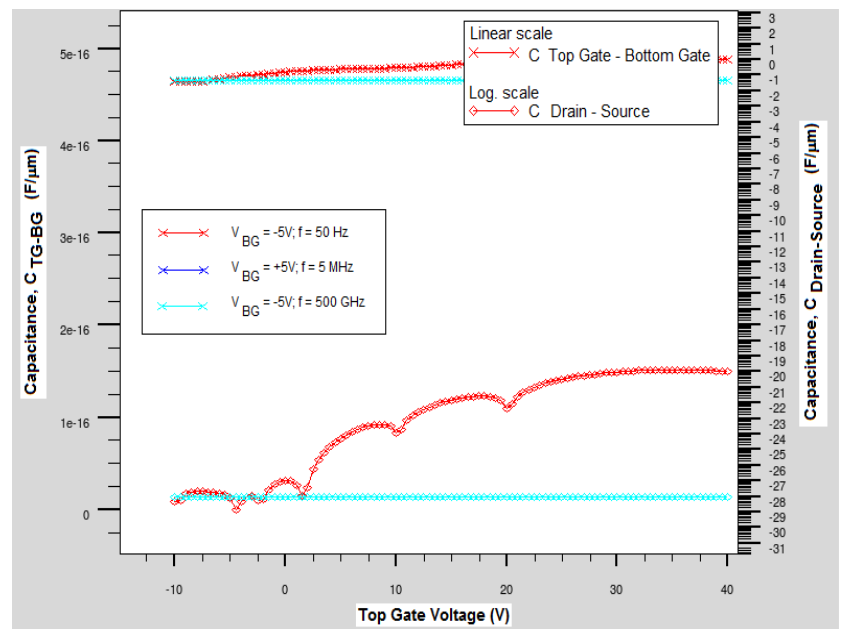

Figure 8. The $C$-V curves at $L F$ and $H F$ for $-10 \mathrm{~V}<V_{T G}<+40 \mathrm{~V}: C_{T G-B G}, C_{D S}$.

Considering the $\mathrm{Oz}$ depth of $5 \mu \mathrm{m}$, the capacitance $\mathrm{C}_{\mathrm{TG}-\mathrm{BG}}=$ $5 \times 10^{-16} \mathrm{~F} / \mu \mathrm{m}$ from fig. 7 , means $10^{-16} \mathrm{~F}$, expressed in Farads. The conductance $G$ reveals a distinct behavior during the frequency rising: $\mathrm{G}_{\mathrm{TG} \text { - BG }}$ suffers a weak variation from $10^{-15} \mathrm{~S}$ to $10^{-14} \mathrm{~S}$, while $\mathrm{G}_{\mathrm{DS}}$ presents degradation from $10^{-15} \mathrm{~S}$ to $10^{-26} \mathrm{~S}$ that means a high resistivity of $10^{26} \Omega$ for $\mathrm{f}>100 \mathrm{~Hz}$, accompanied by current capability degradation at high frequencies.

In a second analysis, the capacitance is traced during the top gate voltage scanning, keeping a constant frequency. Initially the $\mathrm{DC}$ bias is $\mathrm{V}_{\mathrm{S}}=0 \mathrm{~V}, \mathrm{~V}_{\mathrm{D}}=+20 \mathrm{~V}, \mathrm{~V}_{\mathrm{BG}}=-5 \mathrm{~V}$ and $\mathrm{V}_{\mathrm{FG}}=-10 \mathrm{~V}$. Then an AC signal is applied, during the top gate voltage ramping from $-10 \mathrm{~V}$ to $+40 \mathrm{~V}$ to ensure the depletion-enhancement transition, fig. 8. The drain-source capacitance, $\mathrm{C}_{\mathrm{DS}}$, sensitively increases with the voltage from $10^{-28}$ up to $10^{-20} \mathrm{~F} / \mu \mathrm{m}$ only at low frequency $(50 \mathrm{~Hz})$ and preserves constant value of $10^{-28} \mathrm{~F} / \mu \mathrm{m}$ at high frequencies $(500 \mathrm{GHz}$ is superposed over $5 \mathrm{MHz}$ ), fig. 8. Fortunately, the main dynamic parameter, the top gate - bottom gate capacitance, $\mathrm{C}_{\mathrm{TG}-\mathrm{BG}}$, has roughly constant values between $4.5 \div 5 \mathrm{~F} / \mu \mathrm{m}$.

\section{Discussions and Comparisons}

The proposed organic device can be used in different work regimes, in respect with the applied voltages. Because the $\mathrm{n}^{+} \mathrm{p}$ junction has an asymmetrical doping concentration, the conduction thru the longitudinal $\mathrm{n}^{+}$channel prevails.

An interesting behavior occurs for $\mathrm{V}_{\mathrm{TG}}<0, \mathrm{~V}_{\mathrm{BG}}>0$, which allows a longitudinal conduction thru a large neutral n-channel that overlap the metallurgical $\mathrm{n}^{+}$edge and is dominated by an accumulation bottom $n$-channel, near the polyimide interface. The translation of an accumulation electron channel $\left(\mathrm{n} \rightarrow 10^{20} \mathrm{~cm}^{-3}\right.$ near polyimide interface), over the $\mathrm{p}$ region must to be considered under a forward biasing of the vertical $n^{+} p$ junction. In this way, a maximum current peak occurs, $\mathrm{j}_{\mathrm{n} \text {,peak }}=$ $612 \mu \mathrm{A} / \mathrm{cm}^{2}$, fig. 9 . 


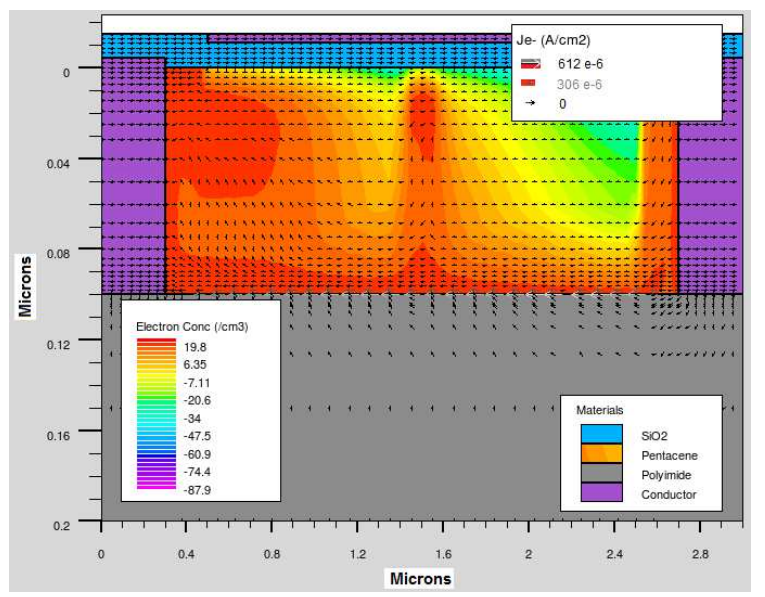

Figure 9. The current vectors through the proposed OFET device and the contours of electrons concentration at $V_{S}=0 \mathrm{~V}, V_{D}=40 \mathrm{~V}, V_{T G}=-10 \mathrm{~V}$ and $V_{B G}=$ $+30 \mathrm{~V}$ - the bottom accumulation channel case.

A positive top gate bias $\left(\mathrm{V}_{\mathrm{TG}}>0\right)$ attracts the electrons near the upper oxide interface (here $\mathrm{n} \rightarrow 10^{20} \mathrm{~cm}^{-3}$ ), offering the maximum drain current densities. The carriers manipulation from the bottom gate increases the total current density from $269 \mu \mathrm{A} / \mathrm{cm}^{2}$ for $\mathrm{V}_{\mathrm{BG}}=-30 \mathrm{~V}$ up to $1900 \mu \mathrm{A} / \mathrm{cm}^{2}$ for $\mathrm{V}_{\mathrm{BG}}=+30 \mathrm{~V}$. However, in whatever work regime, the holes current density rests negligible. In a holes favorable regime, (e.g. for $\mathrm{V}_{\mathrm{TG}}>0$, $\mathrm{V}_{\mathrm{BG}}<0$, when $\mathrm{p}>10^{20} \mathrm{~cm}^{-3}$ near the polyimide interface), the holes peak density barely reaches $\mathrm{j}_{\mathrm{p}}=0.168 \mu \mathrm{A} / \mathrm{cm}^{2}$, while the total current density, $j_{\text {tot }}$, is confounded with the electron peak $\mathrm{j}_{\mathrm{n}}=269 \mu \mathrm{A} / \mathrm{cm}^{2}$. If $\mathrm{V}_{\mathrm{TG}}>0$ is accompanied by a negative bottom gate bias, the proposed device presents two accumulation channels: an n-channel near oxide and a p-channel near the polyimide interface. This situation corresponds to a reverse biasing of the vertical $n^{+} p$ junction, with depleted film in the middle of the organic layer. The depletion is the main cause for the half saturated current, $\mathrm{I}_{\text {Dsat }}=50 \mathrm{fA}$, versus the next work regime. If both top and bottom gate voltages are positives, the maximum drain current performances occurred: $\mathrm{j}_{\text {tot }}=1900 \mu \mathrm{A} / \mathrm{cm}^{2}$ and $\mathrm{I}_{\text {Dsat }}=100 \mathrm{fA}$. At a first sight, the positive top voltage aids the electron accumulation onset, while a positive bottom gate has to reduce the p-type channel form the polyimide vicinity. Due to the initial doping concentration asymmetry in $\mathrm{n}^{+} / \mathrm{p}$ regions, the $\mathrm{p}$ type conduction is poor. The effect of both positive voltages is visible in figure 4 . Here the holes concentration is under $10^{-8} \mathrm{~cm}^{-3}$ in the entire organic film. Also, the electrons concentration reaches two peaks near interfaces, but also has significant value across the entire organic film, $\left(>10^{17} \mathrm{~cm}^{-3}\right)$, with consequences on the total drain current.

The applications of the proposed device, biased at $\mathrm{V}_{\mathrm{TG}}>0$, are based on the output characteristics with one order of magnitude superior versus the work regime with $\mathrm{V}_{\mathrm{TG}}<0$, presenting a characteristics saturation under $12 \mathrm{~V}$, fig. 6 .

The operation frequency, $f$, is considered that frequency that produces a stable value for the top-gate / bottom-gate capacitance and source-drain conductance, far away from the cutoff conditions, [21]. The results are comparatively presented in table 2 .

Table 2. The OTFTs device parameters

\begin{tabular}{llll}
\hline Parameters & $\left|\mathbf{V}_{\text {th }}\right|(\mathbf{V})$ & $\mathbf{V}_{\text {Dsat }}(\mathbf{V})$ & $\mathbf{f}(\mathbf{H z})$ \\
\hline Actual OTFT & $1 \ldots 5$ & $\sim 2 \ldots 28$ & 100 \\
Other OTFT & $17 \ldots 33^{[27]}$ & $\sim 24^{[27]}$ & $100 \mathrm{k}^{[34]}$ \\
\hline
\end{tabular}

Table 3. The organic device work regimes

\begin{tabular}{lllll}
\hline TG and BG biases & Work regime & Typical voltages & Static values & $\mathbf{J}_{\text {peak }}\left[\boldsymbol{\mu A} / \mathbf{c m}^{2}\right]$ \\
\hline $\mathrm{V}_{\mathrm{TG}}<0, \mathrm{~V}_{\mathrm{BG}}>0$ & bottom n-channel & $\mathrm{V}_{\mathrm{TG}}=-10, \mathrm{~V}_{\mathrm{BG}}=30 \mathrm{~V}, \mathrm{~V}_{\mathrm{DS}}=40 \mathrm{~V}$ & $\mathrm{I}_{\text {Dsat }}=2 \div 5 \mathrm{fA} \mathrm{V}_{\text {Dsat }}=2 \mathrm{~V}$ & $\mathrm{j}_{\text {tot }} \approx 612$ \\
$\mathrm{~V}_{\mathrm{TG}}<0, \mathrm{~V}_{\mathrm{BG}} \leq 0$ & volume n-channel & $\mathrm{V}_{\mathrm{TG}}=-10, \mathrm{~V}_{\mathrm{BG}}=-20 \mathrm{~V}, \mathrm{~V}_{\mathrm{DS}}=40 \mathrm{~V}$ & $\mathrm{I}_{\mathrm{Dsat}}=2 \div 12 \mathrm{fA} \mathrm{V}_{\text {Dsat }}=4 \mathrm{~V}$ & $\mathrm{j}_{\text {tot }} \approx 1.45$ \\
$\mathrm{~V}_{\mathrm{TG}}>0, \mathrm{~V}_{\mathrm{BG}}<0$ & top n-channel // bottom p-channel & $\mathrm{V}_{\mathrm{TG}}=+1, \mathrm{~V}_{\mathrm{BG}}=-30 \mathrm{~V}, \mathrm{~V}_{\mathrm{DS}}=40 \mathrm{~V}$ & $\mathrm{I}_{\mathrm{Dsat}}=50 \mathrm{fA} \mathrm{V}_{\text {Dsat }}=12 \mathrm{~V}$ & $\mathrm{j}_{\text {too }} \approx 269$ \\
$\mathrm{~V}_{\mathrm{TG}}>0, \mathrm{~V}_{\mathrm{BG}} \geq 0$ & top n-channel // bottom n-channel & $\mathrm{V}_{\mathrm{TG}}=+10, \mathrm{~V}_{\mathrm{BG}}=30 \mathrm{~V}, \mathrm{~V}_{\mathrm{DS}}=40 \mathrm{~V}$ & $\mathrm{I}_{\text {Dsat }}=100 \mathrm{fA}, \mathrm{V}_{\text {Dsat }}=20 \mathrm{~V}$ & $\mathrm{j}_{\text {too }} \approx 1900$ \\
\hline
\end{tabular}

However, the dynamic analysis reveals capacitance or conductance degradation at extreme low frequencies, fewer than $50 \mathrm{~Hz}$, fig. 8 .

The conduction superiority is established by the static characteristics. A simultaneously association between a negative top gate voltage with a positive bottom gate voltage enhances the electrons distribution in a larger cross section of the organic semiconductor film. This evolution is rather observed at a given $\mathrm{V}_{\mathrm{DS}}$ that determines the current flow with maximum densities of $612 \mu \mathrm{A} / \mathrm{cm}^{2}$ near the polyimide interface, fig. 9 .

Table 3 presents the main features of the device in each work regime. Fortunately, the capacitance range is below $1 \mathrm{fF}$ that is more sensitive as for a single gate OTFT case with $25 \mathrm{pF}$, accessing a superior cutoff frequencies, [21].

Application of this device at low frequencies could be interesting in electrophysiology, where the most useful biosignals posses frequencies under $1 \mathrm{~Hz}$, [22]. The proposed organic transistor is suitable for the biomimetic applications, which involve carriers mobility under $0.1 \mathrm{~cm}^{2} / \mathrm{Vs}$, [23]. Other applications of the organic electronics in bioelectronics envisage transducer with organic materials as biocompatible interfaces, [24-26].

The volume channel regime is advantageously, because the main conduction channel avoids oxide/organic semiconductor or polyimide/ organic semiconductor interfaces, which represent a cause of the OFET characteristics degradation, [27-29].

Another possible application could be a sensitive transducer in those sensors that request a current variation without interfaces interferences, [30].

In the case of two accumulation channels the current 
density gets three orders of magnitude higher than in the case of the volume n-channel. From this point of view, the accumulation mode is more suitable for demands in high power applications.

\section{Conclusions}

This paper presented a novel organic thin film transistor as architecture and as work regimes. The main novelties of this paper were: (i) special architecture with vertical $n^{+} p$ junction; (ii) new work regimes with different channels; (iii) discussions of applications.

The device simulations revealed four distinct behaviors, depending on the Top and Bottom Gate voltages. A positive top gate bias attracts the electrons from the $\mathrm{n}^{+}$channel near the upper oxide interface, where $\mathrm{n} \rightarrow 10^{20} \mathrm{~cm}^{-3}$, offering a maximum drain current densities. In a holes favorable regime, $\mathrm{V}_{\mathrm{TG}}>0, \quad \mathrm{~V}_{\mathrm{BG}}<0$, the holes density peak barely reaches $\mathrm{j}_{\mathrm{p}}=0.168 \mu \mathrm{A} / \mathrm{cm}^{2}$, while the total current density, $\mathrm{j}_{\text {tot }}$, is confounded with the electron peak $\mathrm{j}_{\mathrm{n}}=269 \mu \mathrm{A} / \mathrm{cm}^{2}$. If $\mathrm{V}_{\mathrm{TG}}>0$ was accompanied by a negative bottom gate bias, the proposed device presented two accumulation channels: an upper n-channel near oxide and a bottom p-channel near the polyimide interface. When both gate voltages are positives, the maximum drain current performances occurred: $\mathrm{j}_{\text {tot }}=1900 \mu \mathrm{A} / \mathrm{cm}^{2}$ and $\mathrm{I}_{\text {Dsat }}=100 \mathrm{fA}$. Bioelectronics or biomimetics applications benefit from a low saturation voltages and low carriers mobility. Low threshold voltages and low drain saturation voltages are convenient parameters for the low power consumption.

\section{Acknowledgements}

This work was partially supported by POSDRU/89/1.5/S/62557 Project.

\section{References}

[1] Alejandro de la Fuente Vornbrock, Donovan Sung, Hongki Kang, Rungrot Kitsomboonloha, Vivek Subramanian, Fully gravure and ink-jet printed high speed pBTTT organic thin film transistors, Organic Electronics, Volume 11, Issue 12, pp. 2037-2044, 2010.

[2] K. Bock, Polymer electronics systems-Polytronics, Proceedings of IEEE, vol. 93, no. 8, pp. 1400-1406, 2005.

[3] Tariel Ebralidze, Nadia Ebralidze, Giorgi Mumladze, Molecular Aggregations and Anisotropy Photoinduction in Organic Compounds, Optics. Science PG, Vol. 3, No. 4, 2014, pp. 33-36.

[4] Askari Mohammad Bagher, OLED Display Technology, American Journal of Optics and Photonics. Science PG, Vol. 2, No. 3, 2014, pp. 32-36.

[5] Anita Risteska, Kah-Yoong Chan, Thomas D. Anthopoulos, Aad Gordijn, Helmut Stiebig, Masakazu Nakamura, Dietmar Knipp, Designing organic and inorganic ambipolar thin-film transistors and inverters: Theory and experiment, Organic
Electronics, Volume 13, Issue 12, pp. 2816-2824, 2012

[6] Arash Takshi, Alexandros Dimopoulos, and John D. Madden, Simulation of a Low-Voltage Organic Transistor Compatible With Printing Methods, IEEE Transactions on electron devices, vol. 55, no. 1, pp. 276-282, 2008.

[7] Satria Zulkarnaen Bisri, Taishi Takenobu, Yohei Yomogida, Hidekazu Shimotani, Takeshi Yamao, Shu Hotta, and Yoshihiro Iwasa, High Mobility and Luminescent Efficiency in Organic Single-Crystal Light-Emitting Transistors, Adv. Funct. Mater., vol. 19, pp. 1728-1735, 2009.

[8] L.-L. Chua, J. Zaumseil, J.-F. Chang, E.-C. W. Ou, P. K.-H. Ho,H. Sirringhaus, and R. H. Friend, General observation of n-type field effect behavior in organic semiconductors, Nature, vol. 434, no. 7030, pp. 194-199, 2005.

[9] J. Jakabovic, J.Kovac, M.Weis, D.Hasko, R.Srnanek, P.Valent, R.Resel, Preparation and properties of thin parylene layers as the gate dielectrics for organic field effect transistors, Microelectronics Journal, vol. 40, pp. 595- 597, 2009.

[10] Mohamed Mehdi Jatlaoui, Daniela Dragomirescu, Mariano Ercoli, Michael Krämer, Samuel Charlot, Patrick Pons, Hervé Aubert and Robert Plana, Wireless communicating nodes at $60 \mathrm{GHz}$ integrated on flexible substrate for short-distance instrumentation in aeronautics and space, International Journal of Microwave and Wireless Technologies, Volume 4, Spec. Issue 01, pp 109-117, 2012.

[11] C. Ravariu and F. Babarada, Modeling and simulation of special shaped SOI materials for the nanodevices implementation, Hindawi Journal of Nanomaterials, ID 792759, pp. 1-11, July 2011.

[12] Cristian Ravariu. Semiconductor Materials Optimization for A TFET Device with Nothing Region On Insulator, IEEE Transaction on Semiconductor Manufacturing Journal, vol. 26, issue 3, pp. 406-413, Aug. 2013.

[13] ***, Atlas Manual 2012, available at: www.silvaco.com

[14] Franziska D. Fleischli, Katrin Sidler, Michel Schaer, Veronica Savu, Jürgen Brugger,Libero Zuppiroli, The effects of channel length and film microstructure on the performance of pentacene transistors, Organic Electronics, vol. 12, pp. 336-340, 2011.

[15] S. P. Singh, A. Sellinger, A. Dodabalapur, Electron transport in copper phthalocyanines J. Appl. Phys., 107, 044509, 2010.

[16] G. Bersuker, P. Zeitzoff, G. Brown, and H. R. Huff, Dielectrics for future transistors, Materials Today, vol. 7, no. 1, pp. 26-33, 2004.

[17] Burgi, L.; Richards, T. J.; Friend, R. H.; Sirringhaus, H., Close look at charge carrier injection in polymer field-effect transistors, J. Appl.Phys., vol. 94, issue 9, 6129: 1-9, 2003.

[18] Prashant Sonar, Samarendra P. Singh, Yuning Li, Mui Siang Soh, and Ananth Dodabalapur, A Low-Bandgap Diketopyrrolopyrrole-Benzothiadiazole-Based Copolymer for High-Mobility Ambipolar Organic Thin-Film Transistors, Adv. Mater., vol. 22, pp. 5409-5413, 2010.

[19] Stefan Chisca, Ion Sava, Valentina-Elena Musteata, Maria Bruma, Dielectric and conduction properties of polyimide films, IEEE International Semiconductor Conference CAS 2011, Volume: 2, pp. 253 - 256, 2011. 
[20] Jana Zaumseil and Henning Sirringhaus, Electron and Ambipolar Transport in Organic Field-Effect Transistors, Chem. Rev., 107, pp. 1296-1323, 2007.

[21] Hagen Klauk, Organic thin-film transistors, Chem. Soc. Rev., vol. 39, pp. 2643-2666, 2010.

[22] F. Babarada, C. Ravariu, J. Arhip, Electrophysiological Data Processing Using a Dynamic Range Compressor Coupled to a Ten Bits A/D Convertion Port, in Proceedings IEEE-CCP, Lisa OConner Editor, Conference Publishing Services CPS, IEEE Computer Society, pp. 257-262, 2011.

[23] P. Bremner, Y. Liu, M. Samie, G. Dragffy, A. G. Pipe, G. Tempesti, J. Timmis and A. M. Tyrrell, SABRE: a bio-inspired fault-tolerant electronic architecture, Bioinspir. Biomim. IOP, $8016003,2013$.

[24] G. S. Costa, A. M. Salgado, P. R. G. Barrocas, Advances on Using a Bioluminescent Microbial Biosensor to Detect Bioavailable Hg (II) In Real Samples, American Journal of Bioscience and Bioengineering. Science PG, Vol. 1, No. 3, 2013, pp. 44-48.

[25] Mohammad Movassat, Nasser Ashgriz, Bob Cheung, Blast Wave Induced Flows in Semicircular Canals,American Journal of Bioscience and Bioengineering. Science PG, Vol. 3, No. 1, 2015, pp. 1-7.

[26] Yasser M. Riyad, Sergej Naumov, Jan Griebel, Christian Elsner, Ralf Hermann, Katrin R. Siefermann, Bernd Abel, Optical
Switching of Azophenol Derivatives in Solution and in Polymer Thin Films: The Role of Chemical Substitution and Environment, American Journal of Nano Research and Application. Science PG, Special Issue:Advanced Functional Materials. Vol. 2, No. 6-1, 2014, pp. 39-52.

[27] Tae-Jun Ha, Prashant Sonar, Samarendra P. Singh, and Ananth Dodabalapur, Characteristics of High-Performance Ambipolar Organic Field-Effect Transistors Based on a Diketopyrrolopyrrole-Benzothiadiazole Copolymer, IEEE Transactions on electron devices, vol. 59, no. 5, pp. 1494-1500, 2012.

[28] Poornima Mittal, B.Kumar, Y.S.Negi, B.K.Kaushik, R.K.Singh Channel length variation effect on performance parameters of organic field effect transistors, Microelectronics Journal, Volume 43, Issue 12, pp. 985-994, 2012.

[29] Yoshinori Ishikawa, Yasuo Wada, and Toru Toyabe, Origin of characteristics differences between top and bottom contact organic thin film transistors, Journal of applied physics, 107, 053709, 2010.

[30] Na Liu, Yulei Hu, Jianhua Zhang, Jin Cao, Yinchun Liu, Jun Wang, A label-free, organic transistor-based biosensor by introducing electric bias during DNA immobilization, Organic Electronics, vol. 13, pp. 2781-2785, 2012. 